\title{
A Service-Based Platform for Monitoring and Diagnosis of Patients with SAHS Symptoms
}

\author{
Sara Balderas-Díaz \\ University of Granada, Spain \\ sarabd@correo.ugr.es
}

\author{
Kawtar Benghazi \\ University of Granada, Spain \\ benghazi@ugr.es
}

\author{
José Luis Garrido \\ University of Granada, Spain \\ jgarrido@ugr.es
}

\author{
Gabriel \\ Guerrero-Contreras \\ University of Granada, Spain \\ gjguerrero@ugr.es
}

\author{
Elena Miró \\ University of Granada, Spain \\ emiro@ugr.es
}

\begin{abstract}
The Sleep Apnea Hypopnea Syndrome (SAHS) is a symptomatology that affects between $2-5 \%$ of world populations. This syndrome presents serious consequences in daily life of the people that suffer it and its detection requires an analysis in a hospital with specialized professionals and medical equipment. Because of this, long waiting lists exist. In this paper a platform for remotely SAHS monitoring and diagnosis is presented, which aims to reduce waiting lists and provide an alternative to current systems of diagnosis in a more flexible way. The proposal mainly consists of a platform based on the Service Architecture Oriented (SOA) approach and a modular design has been followed in order to facilitate an incremental number of patients (scalability) and add new functionality (extensibility).
\end{abstract}

\section{Categories and Subject Descriptors}

C.1.3 [Other Architecture Styles]: Cellular architecture; H.3.4 [Systems and Software]: User profiles and alert services; J.3 [Life and Medical Sciences]: Health

\section{General Terms}

Design, Human Factors

\section{Keywords}

Wireless and Mobile Computing, Multiple Sensory Devices, Service Oriented Architecture (SOA), eHealth, Sleep Apnea Hipopnea Syndrome (SAHS), Patients Monitoring

\section{INTRODUCTION}

The current economic recession has a direct impact on the lives level of people. Healthcare is one of the sectors most affected by this situation and also one of the most worrying factors for the future [7]. In order to provide a high-quality, accessible and sustainable healthcare system, governments

Permission to make digital or hard copies of all or part of this work for personal or classroom use is granted without fee provided that copies are not made or distributed for profit or commercial advantage and that copies bear this notice and the full citation on the first page. To copy otherwise, to republish, to post on servers or to redistribute to lists, requires prior specific permission and/or a fee. REHAB 2014, May 20-23, Oldenburg, Germany Copyright $\odot 2014$ ICST 978-1-63190-011-2 DOI 10.4108/icst.pervasivehealth.2014.255365 are investing a great deal of resources in research. In this context, new information and communication technologies (ICT) play a key role by providing the capabilities needed to deliver services more efficient, effective, reliable and fast, achieving an improvement in the diagnosis and treatment of patients, reducing waiting times and saving costs [4]. However, the implementation of these new technologies spending is a profitable long-term economic.

In particular, a common problem in the people's daily life is not getting a non-restful sleep. In this case, person may be suffering some sleep disorder from among the more than 90 cases that exist [8]. Specifically, the Sleep Apnea-Hypopnea Syndrome (SAHS) [3] implies high blood pressure, a serious decrease in quality of life, traffic and workplace accidents or even die asleep.

Nowadays, between the $2-5 \%$ of the world's population suffer from this syndrome and from which close to $90-95 \%$ have not been diagnosed. In fact, a long waiting list exists for analysis and diagnosis in hospitals with specialized medical equipment. Usually, for diagnosis of SAHS it is necessary to perform a test in a specialized room called Sleep Room (nocturnal polysomnography) which requires the patient to remain asleep for several hours. Likewise, the patient is in a strange environment so in many cases makes it more difficult for sleeping which requires to, repeat the test with the repercussions that this have on the own patient and on the waiting list. Furthermore, the sleep room has a sophisticated, static, heavy and expensive medical equipment which allows for very detailed studies of patients. Also there are needed medical specialized staff for the placement of some equipment in the body of the patient. These medical staff monitor the patient while he or she sleeps, in case some problem happens in the placement of the equipment or in case the patient needs an urgent medical attention because he/she is in a critical state. At present the nocturnal polysomnography is the most reliable study used to detect whether a person suffers from this disease [2].

Nowadays, it is important the good acceptance that the new technologies are having between the population, the evolution in mobile devices allows to create portable systems, with low economic cost and wireless connections, therefore, the distribution of the system among users is more feasible, it is possible to dispose more units for the same price and it is 
more comfortable to use, due to the reduced wiring and connections between system elements. Thus medical specialists can realize a diagnosis and monitoring of the patient's status, as well as to specify a treatment and to control his/her evolution. Furthermore, the tests can be repeated as many times as necessary, owing to availability and low cost of the equipment. Moreover, the patient could be in a known environment e.g.(at home) and then more comfortable, which may help to the effectiveness of test performed.

In this paper, a platform for remote monitoring and diagnosis is proposed. This platform aims facilitate the analysis, monitoring and diagnosis of patients who could suffer the sleep apnea-hypopnea syndrome. It makes possible to reach to a major number of persons at the same time, to offer a more attainable service to persons who have certain difficulties, for example, elderly people or persons with special needs, who could have difficulties to move about to medical centre, in addition this could reduce the long waiting-lists for the test of polysomnography.

The rest of this paper is structured as follows. Section 2 presents some related work. In Section 3, the platform for monitoring and diagnosis of patients with SAHS symptoms is presented. Finally, conclusions and future work are summarized in Section 4.

\section{RELATED WORK}

The positive acceptance of technology among the population, favours the appearance of new health care systems, which are intended to improve the daily life of the patients. Such as systems have great potential, since can they offer new functionalities and provide support to medical specialists, patients and families [6]. There are currently several platforms for remote monitoring of patients at home. Hygehos Home [1] allows patient to send medical information (weight, blood glucose level, etc.) from their own home and it can control whether the patient takes the medication indicated by the specialist. In [10] is proposed intelligent selfadjusting pillow for detecting and perform an apnea treatment. For detecting sleep apnea a blood oxygen sensor is used and according to the parameters captured by that sensor, the pillow is adjusted automatically both in height and in the form. In [9] is presented a platform for remote monitoring of patients with brain injury. The system allows carry out a track of daily activities that the patient perform and autonomously to do rehabilitation exercises.

However, most of this works do not deal directly with the problems of sleep apnea syndrome, and jobs that focus on this disease, only provide local mechanisms to try to mitigate their symptoms. This proposal aims to develop a system of remote diagnostic for sleep apnea, in order to facilitate and to expedite the work of specialists and provide comfort to patients.

\section{PLATFORM FOR MONITORING AND DIAGNOSIS OF PATIENTS WITH SAHS SYMPTOMS}

In this section a service-based platform for monitoring and diagnosis of patients is presented. The objective is to provide a health system with high-quality services, this is, it offers the possibility to realize a continued monitoring, reducing costs to allow repeat test as many times as necessary; establish a more in-depth contact between patient and medical specialists; and improve the patients' quality of life, due to they feel cared for and supported, and thus more calm about their health.

This platform is based on the service-oriented architecture (SOA) paradigm to guarantee interoperability, platform independence and reusability, among others. Figure 1 shows the architecture of the proposed platform. A modular design approach has been followed in order to facilitate scalability in terms of numbers of patients who can use the system, and functional extensibility.

The platform is made up of three subsystems which make use of two main services. The three subsystems cooperate among each other: (1) monitoring subsystem, which is located at patient's home. The patient makes use of a medical equipment (biosensors) that he/she should place in his/her own body. The sensors act as nodes that should be strategically positioned to capture the medical data and communicate among themselves. In this way, monitoring system use emerging wearable wireless body area networks (WBANs), following one of the most promising approaches [5]. (2) A subsystem for a relative, which receives information from monitoring subsystem about patient's status, in order to allow a collaborative supervision from relative. This relative can be at home or at patient's house. Finally, (3) medical subsystem, which allows to medical specialist to supervise the patient from his or her workplace or access the reports generated from the conducted studies. Both, medical specialist and family member can access to these information trough of a mobile device. Therefore, the platform design ensures, through of different subsystems, an intuitive and customizable environment for different users and devices that share information.

Two main services has been developed to give support for these subsystems: (1) "Database Management" service is responsible of storing the information about patients and provides a query service. Furthermore, this service acts like an intermediate layer for security purposes, since it provides restriction mechanisms in data access and modification. (2) "Patient Information Management" service provides complex information through processing of the basic information, in order to reduce the workload of mobile devices and to allow code reusability, the same information to be accessed by different subsystems. It is important to mention that these services can be deployed in a local server, which ensures information control, or on the contrary, in a cloud infrastructure, which provides higher information accessibility. These components are discussed in more detail in the next section.

\subsection{Platform Subsystems}

- Patient Monitoring. The objective of the Patient Monitoring subsystem is to emulate the sleep room of the medical centre. It is made up of biosensors and a central component. This central component is a gateway which receives and filters the data from the sensors and sends it to Database Management service. Moreover, if a disconnection occurs during the monitoring session, and it has no connection with the service, it 


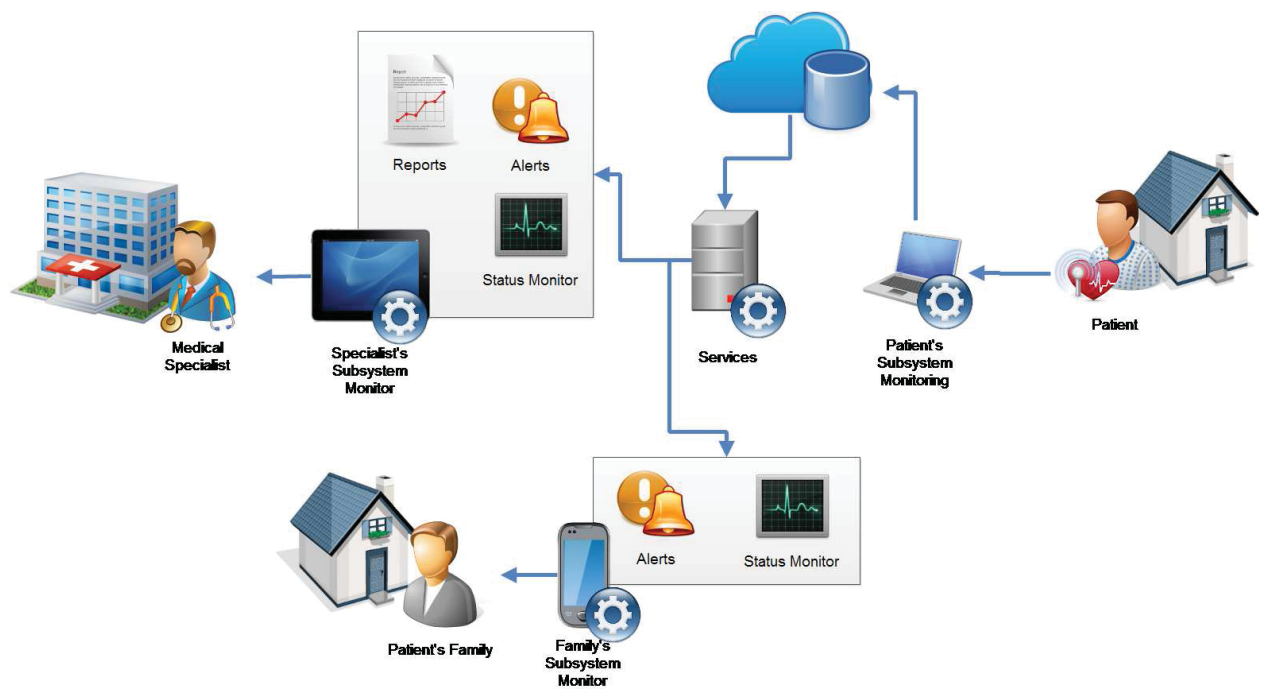

Figure 1: Monitoring and diagnosis platform architecture.
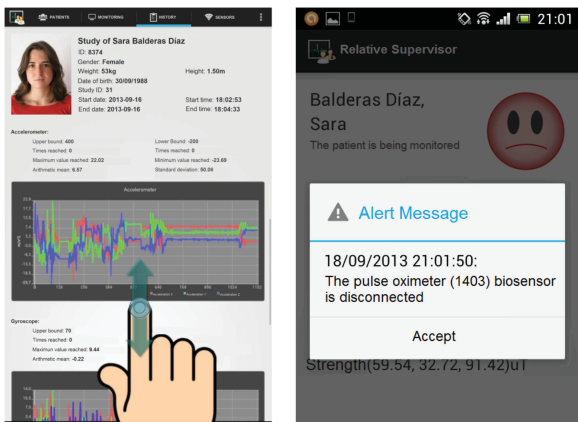

Figure 2: (Left) Example of a study performed to a patient. (Right) Example of an alert message in "Relative Supervision Subsystem" application.

stores the information collected locally. This central component can be the patient's computer or an embedded system specifically designed for this task.

Regarding to the biosensors, patient should learn to use them before take them home. When he or she goes to sleep, biosensors must be connected, then, the monitoring session may be started. It must be emphasized that these biosensors have a lower cost that biosensor used in sleep room. This allows that the test may be repeated as many times as necessary and without causing delays in waiting lists. The platform makes use of wireless and ergonomics devices to guarantee patients' comfort and not unduly interfere his or her sleep.

- Specialist Supervisor. Through the subsystem Specialist Supervisor, the medical specialist can monitor the patient in real time, consult the reports generated from the studies conducted (Figure 2, left), access the patient's personal data and manage biosensors registered in the system; it is possible to assign/unassign biosensors to patients in real time and remotely (from his or her workplace). Note that this allows to specialist to monitor more than one patient at a time, without having to visit each patient's location.

A study of the patient's sleep contains the following information: date and time of procurement (start and end), patient weight and height, and for each biosensor connected to patient: maximum and minimum value reached during the study, upper and lower limits specified by the specialist, times reached, average, standard deviation and an interactive graph. This view is dynamically generated for each study depending on biosensors used and the profile of each patient.

Additionally, the medical specialist can activate separate independents alarms for each biosensor. He or she can adjust alarm values (maximum and minimum limits), and if a limit is exceeded the system will send an audible notification to the specialist. This notification contains information about the patient who is in danger situation and the threshold value which has been exceeded. While this situation continues, the notification will repeat periodically. Besides, volume and sound type can be customized, and these notifications can be enabled or disabled in general.

Finally, for more flexibility, these functionalities are available via a mobile device, such a tablet. This facilitates that this information to be accessed by a group of specialists, who are working together collaboratively.

- Relative Supervisor. The Relative Supervisor subsystem allows relatives of the patient to collaborate in of him or her monitoring. This subsystem operates on the mobile device of the patient's family and allows to him or her to monitor the patient condition in real time.

The subsystem displays the values captured by the biosensors and indicates if patient's condition is normal or a condition of risk has been detected (Figure 2, right). Also, it has an alerts system connected to specialist system, if the values captured by the biosensors 
exceed the values established by the specialists, the platform sends an audible notification to the patient's family, which allows the patient's family to react to a critical case.

\subsection{Platform Services}

- Database Management. This service provides an abstraction layer between data and application, offering functionalities that allow manage data at high level, reducing the efforts of the developers. The service guarantees the security in access and management to external databases, since that all connections are performed through it, which will be responsible of aspects of configuration and permissions of access. Moreover, standards of web services, such as SOAP and WSDL, are being used for uniform access and to provide independence from platform, in this way, different devices with different properties, at hardware and software level, can access to this service. Furthermore, owing to design services is allowed interoperability with other systems, services or different applications, i.e, it is allowed exchange and access to information of other independent systems, services or applications.

- Patient Information Management. This service allows to perform a processing of the information with objective to provide complex information that may require an intensive computing. Operation is as follows, this service receives a request of an application, it processes the request and determines the basic queries that compose it. Then, it is contacted with the Database Management Service, which sends the requested information, and the Patient Information Management Service processes the information received in order to obtaining the request high level information. As in the previous case, this service also ensures security, interoperability and platform independence.

\section{CONCLUSIONS AND FUTURE WORK}

In this paper a service platform has been presented. This platform enables remote monitoring and diagnosis of patients with possible symptoms of SAHS. The platform has been developed with the aim to improve health care for patients. With the use of low-cost devices, the system that has been created allows monitoring of patients in their own homes, while the specialist in charge of treating them is in the hospital. This platform is proposed as a possible alternative to other detection methods currently implemented. Thus, it aims to reduce waiting lists, that can reach up to two years, and improve health and quality of life of patients. The proposal avoids the movement of patients to the hospital, promoting better and faster patient recovery, economic savings in the health system and greater flexibility in the management and care of patients.

The platform has been developed under the approach of service-oriented architecture (SOA), providing advantages such as platform independence, scalability, reusability and autonomy. Finally, the platform has a modular design to facilitate scalability and allow adding new biosensors and new functionality easily in the future.
As future work, it is intended to extend diagnostic system, with the objective of providing to medical specialist a better support, and expand alert system with context-awareness capabilities. Moreover, it is intended to perform tests with real patients.

\section{ACKNOWLEDGMENT}

This research has been partially supported by the Spanish Ministry of Economy and Competitiveness with European Regional Development Funds (FEDER) under the research project TIN2012-38600 and by the Granada Excellence Network of Innovation Laboratories (GENIL) under project PYR-2014-5. The authors would also like to acknowledge contribution from COST Action AAPELE1303.

\section{REFERENCES}

[1] Hygehos home web, 05-02-2014. http://www.hygehos.com/.

[2] A. L. Chesson Jr, R. A. Ferber, J. M. Fry, M. Grigg-Damberger, K. M. Hartse, T. D. Hurwitz, S. Johnson, G. A. Kader, M. Littner, G. Rosen, et al. The indications for polysomnography and related procedures. Sleep, 20(6):423-487, 1997.

[3] G. Gould, K. Whyte, G. Rhind, M. Airlie, J. Catterall, C. Shapiro, and N. Douglas. The sleep hypopnea syndrome. American Review of Respiratory Disease, 137(4):895-898, 1988.

[4] J. W. Hill and P. Powell. The national healthcare crisis: Is ehealth a key solution? Business Horizons, 52(3):265-277, 2009.

[5] E. Jovanov, A. Milenkovic, C. Otto, and P. C. De Groen. A wireless body area network of intelligent motion sensors for computer assisted physical rehabilitation. Journal of NeuroEngineering and rehabilitation, 2(1):6, 2005.

[6] G. L. Kreps and L. Neuhauser. New directions in ehealth communication: Opportunities and challenges. Patient Education and Counseling, 78(3):329-336, 2010.

[7] C. Lionis and E. Petelos. The impact of the financial crisis on the quality of care in primary care: an issue that requires prompt attention. Quality in primary care, 21(5):269, 2013.

[8] A. A. of Sleep Medicine et al. International classification of sleep disorders: diagnostic and coding manual, 2005.

[9] A. Ruiz-Zafra, M. Noguera, K. Benghazi, J. L. Garrido, G. C. Urbano, and A. Caracuel. A mobile cloud-supported e-rehabilitation platform for brain-injured patients.

[10] J. Zhang, Q. Zhang, Y. Wang, and C. Qiu. A real-time auto-adjustable smart pillow system for sleep apnea detection and treatment. In Proceedings of the 12th international conference on Information processing in sensor networks, pages 179-190. ACM, 2013. 